\title{
MicroRNA-137 regulates hypoxia-mediated migration and epithelial-mesenchymal transition in prostate cancer by targeting LGR4 via the EGFR/ERK signaling pathway
}

\author{
HAO ZHANG ${ }^{1}$, FANG LIANG $^{2}$, JUNMIN YUE ${ }^{1}$, PENG LIU $^{1}$, JUNYONG WANG ${ }^{1}$, ZHAOYANG WANG $^{1}$, \\ HONGXING LI ${ }^{1}$, DUO CHENG ${ }^{2}$, JIE DU ${ }^{2}$, KAI ZHANG ${ }^{1}$ and PENG DU ${ }^{3}$
}

\begin{abstract}
Departments of ${ }^{1}$ Urology and ${ }^{2}$ Oncology, Zhengzhou Central Hospital Affiliated to Zhengzhou University (Zhengzhou Central Hospital), Zhengzhou, Henan 450007; ${ }^{3}$ Department of Urology, Key Laboratory of Carcinogenesis and Translational

Research (Ministry of Education/Beijing ), Peking University Cancer Hospital and Institute, Beijing 100142, P.R. China
\end{abstract}

Received June 12, 2019; Accepted April 15, 2020

DOI: 10.3892/ijo.2020.5064

\begin{abstract}
MicroRNAs (miRs) serve an integral role in prostate cancer. The present study aimed to investigate the effects and mechanisms of miR-137 in hypoxia-mediated migration and epithelial-mesenchymal transition (EMT). PC3 and DU145 prostate cancer cells were exposed to hypoxia for $24 \mathrm{~h}$, after which the expression of miR-137 was determined by reverse transcription-quantitative PCR (RT-qPCR). The cells were transfected with a miR-137 mimic or inhibitor, followed by hypoxia exposure. The results demonstrated that hypoxia reduced miR-137 expression. Further results from the Cell Counting Kit-8, Cell Death Detection ELISA plus kit, Transwell assay, RT-qPCR and western blotting assays revealed that the miR-137 mimic prevented cell proliferation, facilitated apoptosis and repressed cell migration, invasiveness, and expression of $\mathrm{N}$-cadherin, vimentin and matrix metalloproteinase 2; the miR-137 inhibitor exerted the opposite effects. A dualluciferase reporter assay determined that miR-137 directly targeted leucine-rich repeat-containing $\mathrm{G}$ protein-coupled receptor 4 (LGR4). Additionally, miR-137 negatively regulated the epidermal growth factor receptor/extracellular signalregulated kinase (EGFR/ERK) signaling pathway by targeting LGR4. LGR4 silencing or EGFR/ERK inhibition abolished the effects of miR-137 inhibitor on cell migration and EMT. In conclusion, by targeting LGR4 via the EGFR/ERK signaling pathway, miR-137 inhibited prostate cancer cell migration and EMT.
\end{abstract}

Correspondence to: Dr Hao Zhang, Department of Urology, Zhengzhou Central Hospital Affiliated to Zhengzhou University (Zhengzhou Central Hospital), 195 Tongbai Road, Zhongyuan, Zhengzhou, Henan 450007, P.R. China

E-mail: haozhang_uro@163.com

Key words: miR-137, migration, epithelial-mesenchymal transition, EGFR/ERK, LGR4, prostate cancer

\section{Introduction}

Prostate cancer is the most common malignant tumor among men in developed countries (1). In recent years, prostate cancer has contributed to a high morbidity and mortality in most native Asian populations, with $>297,000$ new prostate cancer cases diagnosed in Asia, accounting for $23.3 \%$ of the global incidence and $\sim 33 \%$ of deaths occurring in Asia in $2018(2,3)$. Currently, prostate gland removal and androgen blockade is an effective treatment option for the majority of patients with prostate cancer; however, a number of patients experience hormone resistance, and prostatectomy is dangerous for older patients with cardiovascular disease (4). Hypoxia is a common biological feature of solid tumors, usually associated with cancer progression, treatment resistance, and poor prognosis (5). Hypoxia reportedly triggers expression of adherens junction molecules to promote the invasion and epithelial-mesenchymal transition (EMT) of prostate cancer cells $(6,7)$. Therefore, it is necessary to elucidate the molecular mechanisms of prostate cancer progression and explore novel targets for regulating its progression under hypoxic conditions.

MicroRNAs (miRs) serve complicated roles in pathophysiological tumor processes; for example, miR-150 regulates TRPM4-mediated $\beta$-catenin pathway to impede the progression of prostate cancer (8-10). Additionally, miRs serve as prognostic markers of tumors or potential therapeutic targets $(11,12)$. miR-383-5p has been demonstrated to be downregulated in lung adenocarcinoma tissues and to be associated with tumor size and differentiation; in addition, miR-383-5p exerts an antiproliferative function, suggesting that it may serve as a potential novel potential prognostic biomarker and therapeutic target for lung adenocarcinoma (13). Aberrant expression of miR is associated with dysregulation of cancer cell proliferation, apoptosis, and migration. For example, miR-181a and miR-483-5p are overexpressed in prostate cancer, leading to prostate cancer growth $(14,15)$. However, miR-129 is down-regulated in prostate cancer tissues and cell lines, and restoring miR-129 expression reduces the viability, proliferation, and migration of PC3 cells (16). A recent study has demonstrated that miR-150 inhibits EMT in prostate 
cancer cells (10). Patients with prostate cancer recurrence following radical prostatectomy present with reduced miR-137 in prostatectomy specimens (17). Li et al (18) have reported that miR-137 expression is decreased in the mouse brain under hypoxic conditions. However, the potential roles of miR-137 in hypoxia-mediated migration and EMT of prostate cancer cells remain unclear.

The aim of the present study was to clarify the function of miR-137 in prostate cancer cell migration, invasion and EMT, as well as to elucidate the underlying mechanism under hypoxic conditions in prostate cancer cells. This may help determine whether miR-137 may serve as a potential therapeutic target for the treatment of prostate cancer and provide a new mechanism of hypoxia-induced migration, invasion and EMT.

\section{Materials and methods}

Cell culture. PC3 and DU145 cells were obtained from the American Type Culture Collection (ATCC, Rockville, MD, USA). The cells were cultured in Dulbecco's Modified Eagle's medium (DMEM; Gibco; Thermo Fisher Scientific, Inc.) supplemented with $10 \%$ fetal bovine serum (HyClone; Cytiva) in an incubator containing $95 \%$ air and $5 \% \mathrm{CO}_{2}$ at $37^{\circ} \mathrm{C}$.

Cell transfection and hypoxia treatment. The miR-137 mimic, miR-137 inhibitor, and miR-137 negative control (NC) were designed by and synthetized from Shanghai GenePharma Co., Ltd. Leucine-rich repeat-containing $G$ protein-coupled receptor (4LGR4)-specific siRNA (siLGR4) and the siRNA control (siCtrl) were obtained from Shanghai GenePharma Co., Ltd. PC3 and DU145 cells $\left(2.5 \times 10^{5}\right.$ cells/well $)$ were seeded in 6-well plates overnight and transfected with 50 pmol $\mathrm{NC}$, miR-137 mimic or miR-137 inhibitor using Lipofectamine ${ }^{\circledR}$ 2000 (Invitrogen; Thermo Fisher Scientific, Inc.), as well as 50 pmol siLGR4. The sequences used were as follows: miR-137 mimic, 5'-UUAUUGCUUAAGAAUACGCGUAG-3'; miR-137 inhibitor, 5'-CUACGCGUAUUCUUAAGCAAUAA-3'; NC for miR-137, 5'-UCACAACCUCCUAGAAAGAGUAGA-3'; siLGR4, 5'-GGUAAGAAACUCCUAAUUAUU-3'; siCtrl, 5'-TTCTCCGAACGTGTCACGT-3'. Cells were cultured for 4-6 h at $37^{\circ} \mathrm{C}$, and the medium was replaced with complete DMEM. At $48 \mathrm{~h}$ post-transfection, cells were exposed to $24-\mathrm{h}$ hypoxia $\left(\mathrm{H} ; 1 \% \mathrm{O}_{2}, 5 \% \mathrm{CO}_{2}\right.$ and $\left.94 \% \mathrm{~N}_{2}\right)$ in an incubator (BioSpherix, Ltd.). For epidermal growth factor receptor (EGFR) inhibition, $100 \mathrm{nM}$ AG1478 (EMD Millipore) was added to the cells $1 \mathrm{~h}$ prior to hypoxia.

Cell viability analysis. The viability of PC3 and DU145 cells was detected using the Cell Counting Kit-8 (CCK-8) assay

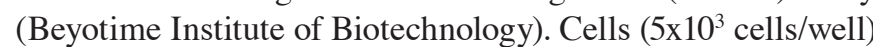
were seeded in a 96-well plate, transfected with the miR-137 mimic or inhibitor and exposed to hypoxia. CCK-8 reagent was added to the cells $(10 \mu \mathrm{l} /$ well $)$ and incubated for $2 \mathrm{~h}$ at $37^{\circ} \mathrm{C}$. The absorbance of each well was recorded at $450 \mathrm{~nm}$ by a microplate reader.

Apoptosis assessment. The Cell Death Detection ELISA PLUS kit (Roche Diagnostics, GmbH) was used to evaluate the apoptosis of DU145 and PC3 cells according to the manufacturer's instructions. In brief, transfected cells $\left(1 \times 10^{4}\right.$ cells/well $)$ were seeded in 96-well plates and subjected to hypoxic conditions. The cells were lysed and centrifuged, and the supernatant was transferred into a streptavidin-coated microplate and incubated with the immunoreagent for $2 \mathrm{~h}$ at room temperature. Absorbance of each well was recorded at $405 \mathrm{~nm}$.

Transwell assay. A Transwell assay was used to measure migration and invasion of PC3 and DU145 cells. For the migration assay, cells $\left(1.5 \times 10^{5}\right.$ cells/well $)$ were seeded in serum-free DMEM and plated into the upper chamber of a 24-well Transwell plate with $8.0-\mu \mathrm{m}$ pores (BD Biosciences). Subsequently, $600 \mu \mathrm{l}$ DMEM supplemented with $20 \%$ fetal bovine serum was added to the lower chamber. After 24-h incubation at $37^{\circ} \mathrm{C}$, the medium was discarded, and the cells were washed with phosphate-buffered saline and fixed with $4 \%$ paraformaldehyde at room temperature for $30 \mathrm{~min}$. The cells were stained with $0.1 \%$ crystal violet at room temperature for $20 \mathrm{~min}$. Images of cells in five random fields (magnification, $\mathrm{x} 200$ ) were captured and the cells were counted under an optical inverted microscope. For the invasion assay, the cells $\left(2.5 \times 10^{5}\right.$ cells/well $)$ were seeded into the upper chamber of a Transwell plate pre-coated with Matrigel (BD Biosciences) at $37^{\circ} \mathrm{C}$ for $30 \mathrm{~min}$, and the protocol was the same as that of the migration assay.

Reverse transcription-quantitative PCR (RT-qPCR). Total RNA of PC3 and DU145 cells was extracted using TRIzol ${ }^{\circledR}$ reagent (Invitrogen; Thermo Fisher Scientific, Inc.) according to the manufacturer's protocol. A spectrophotometer (Thermo Fisher Scientific) was used to quantify the concentration of RNA. A PrimeScript II 1st strand cDNA Synthesis Kit (Takara Bio, Inc.) was used to reverse-transcribe the RNA into cDNA at $42^{\circ} \mathrm{C}$ for $60 \mathrm{~min}$ and $70^{\circ} \mathrm{C}$ for $5 \mathrm{~min}$. Quantitative PCR was performed with an iQ SYBR ${ }^{\circledR}$ Green Super Mix (BioRad Laboratories, Inc.) on an ABI PRISM 7000 Fluorescent Quantitative PCR System (Applied Biosystems, Inc.). The thermocycling conditions were as follows: $95^{\circ} \mathrm{C}$ for $5 \mathrm{~min}$; followed by 40 cycles of $95^{\circ} \mathrm{C}$ for $5 \mathrm{sec}$ and $60^{\circ} \mathrm{C}$ for $30 \mathrm{sec}$. U6 or GAPDH was used as the internal control, and the expression levels of miR-137 or mRNAs were quantified using the $2^{-\Delta \Delta \mathrm{Cq}}$ method (19). The primer sequences were as follows: miR-137 forward, 5'-TATTGCTTAAGAATACGCGTA G-3' and reverse, 5'-AACTCCAGCAGGACCATGTGAT-3'; U6 forward, 5'-CTCGCTTCGGCAGCACA-3' and reverse, 5'-AACGCT TCACGA ATT TGCGT-3'; LGR4 forward, 5'-CTTTGTTTGCCATTTCCTA-3' and reverse, 5'-CTAGTG AGTTTAATAGCACTAA-3'; N-cadherin forward, 5'-CAT CCCTCCAATCAACTTGC-3' and reverse, 5'-ATGTGCCCT CAAATGAAACC-3'; Vimentin forward, 5'-TGTCCAAAT CGATGTGGATGTTTC-3' and reverse, 5'-TTGTACCATTCT TCTGCCTCCTG-3'; MMP2 forward, 5'-TCTTCAAGGACC GGTTCATTTG-3' and reverse, 5'-GATGCTTCCAAACTT CACGCTC-3'; GAPDH forward, 5'-GGAGCGAGATCCCTC CAAAAT-3' and reverse, 5'-GGCTGTTGTCATACTTCT CATGG-3'.

Western blotting. Total protein was collected using a RIPA lysis buffer (Beyotime Institute of Biotechnology) according to manufacturer's protocol. The concentration of each sample 
was determined by BCA Protein Assay kit (Beyotime Institute of Biotechnology). Equal amounts of protein from each sample (50 $\mu \mathrm{g} /$ lane) were separated by 6 or $10 \%$ SDS-PAGE and transferred to a polyvinylidene fluoride membrane. The membrane was blocked with $5 \%$ bovine serum albumin (Amresco, LLC) and then incubated with primary antibodies against LGR4 (cat. no. ab137480; 1:1,000), N-cadherin (cat. no. ab76057; 1:1,000), vimentin (cat. no. ab92547; 1:1,000; all from Abcam), MMP2 (cat. no. 40994; 1:1,000), phosphorylated (p)-EGFR (cat. no. 3777; 1:1,000), EGFR (cat. no. 4267; 1:1,000), p-ERK (cat. no. 4370; 1:1,000), ERK (cat. no. 4695; 1:1,000; all from Cell Signaling Technology, Inc.), $\beta$-actin (cat. no. sc-58673; $1: 5,000$ ) and GAPDH (cat. no. sc-47724; 1:5,000; both from Santa Cruz Biotechnology, Inc.) overnight at $4^{\circ} \mathrm{C}$. The membranes were washed with $0.1 \%$ Tween in TBS (Boster Biological Technology) and incubated with horseradish peroxidase-conjugated goat anti-rabbit IgG (cat. no. ZDR-5306; 1:10,000) or goat anti-mouse IgG (cat. no. ZDR-5307; 1:10,000; both from OriGene Technologies, Inc.) secondary antibodies for $1 \mathrm{~h}$ at room temperature. Pierce Enhanced chemiluminescence Western Blotting Substrate (Thermo Fisher Scientific, Inc.) was used to visualize the protein bands, and the optical density of the bands was analyzed using ImageJ version 1.8.0 software (National Institutes of Health). The relative expression of the proteins was measured using $\beta$-actin or GAPDH as an internal control.

Dual-luciferase reporter gene assay. Two bioinformatics software programs, miRDB (http://mirdb.org/) and microRNA. org (http://www.microrna.org/microrna/home.do), were used to determine whether LGR4 was a target gene of miR-137. The wild-type (wt) 3'-untranslated region (UTR) of LGR4 was amplified and cloned into a pMiR-reporter vector (Promega Corporation). A mutation site of LGR4 in a seed sequence was generated and inserted into the pMiR-reporter vector (mut-LGR4-3'-UTR). The LGR4 3'-UTR was sequenced, and the nucleotide alignment was compared with that in the NCBI database (https://blast.ncbi.nlm.nih.gov/Blast.cgi; NM_018490.5). PC3 cells $\left(4 \times 10^{4}\right.$ cells/well) were seeded into a 24-well plate and co-transfected with miR-137 mimic or NC and wt or mut-LGR4-3'-UTR. After 48-h transfection, a Dual-Luciferase Reporter assay (Promega Corporation) was conducted to analyze the firefly and Renilla luciferase activities according to the manufacturer's instructions. Firefly luciferase activity was normalized to Renilla luciferase activity.

Statistical analysis. All experiments were repeated at least three times. Data are presented as the mean \pm SD and were analyzed using SPSS 11.0 software (SPSS, Inc.). One-way analysis of variance followed by Bonferroni post hoc test was used to compare the differences among multiple groups. $\mathrm{P}<0.05$ was considered to indicate a statistically significant difference.

\section{Results}

Hypoxia down-regulates miR-137 expression in prostate cancer cells. The expression of miR-137 was determined in prostate cancer cells under hypoxic conditions. PC3 and DU145 cells were selected as they have been commonly used to study prostate cancer progression and exhibit high levels of migratory and invasive activity $(20,21)$. As presented in Fig. 1A, PC3 and DU145 cells exposed to hypoxia exhibited a decrease in miR-137. The results of RT-qPCR also demonstrated high miR-137 levels following transfection with the miR-137 mimic and low miR-137 levels following transfection with the miR-137 inhibitor compared with those transfected with the NC in PC3 and DU145 cells (Fig. 1B and C).

miR-137 affects prostate cancer cell viability and apoptosis. Cell viability and apoptosis of PC3 and DU145 cells were assayed. The results demonstrated that hypoxia induced cell proliferation, transfection with the miR-137 mimic reduced cell viability, and the miR-137 inhibitor promoted the survival of PC3 cells compared with the NC group (Fig. 2A). Hypoxia had no effect on apoptosis; however, miR-137 positively regulated apoptosis compared with the NC-transfected PC3 cells (Fig. 2B). Similar trends were observed in DU145 cells, as hypoxia enhanced cell viability, but did not affect apoptosis, whereas the miR-137 mimic reduced cell viability and facilitated apoptosis, and inhibition of miR-137 promoted cell survival and decreased apoptosis (Fig. 2C and D). These results indicated that miR-137 regulated the viability and apoptosis of PC3 and DU145 cells.

miR-137 inhibits cell migration and invasion under hypoxia. PC3 and DU145 cells were exposed to hypoxia after transfection with the miR-137 mimic or inhibitor. Cell migration and invasion were assessed using Transwell assays. The miR-137 mimic inhibited hypoxia-induced cell migration (Fig. 3A) and invasion (Fig. 3B) compared with those in the $\mathrm{NC}+\mathrm{H}$ group. Inhibition of miR-137 displayed the opposite results in PC3 cells. In addition, the migration (Fig. 3C) and invasion (Fig. 3D) of DU145 cells were inhibited by the miR-137 mimic and promoted by miR-137 inhibitor. These results indicated that miR-137 blocked the hypoxia-induced migration and invasion of prostate cancer cells.

miR-137 suppresses the hypoxia-induced EMT. To investigate how miR-137 affects EMT in prostate cancer cells, images of cell morphology were captured, and it was observed that hypoxia induced a fusiform morphology; the miR-137 mimic reversed this effect, whereas the miR-137 inhibitor further induced the EMT morphology of PC3 cells compared with the NC-transfected cells (Fig. S1). Similarly, in DU145 cells, the miR-137 mimic inhibited the hypoxia-mediated EMT morphology, however, no differences were observed in cell morphology between the $\mathrm{NC}+\mathrm{H}$ and inhibitor $+\mathrm{H}$ groups (Fig. S1). In order to evaluate whether EMT occurred in PC3 and DU145 cells after transfection with the miR-137 mimic or inhibitor and hypoxia, the expression levels of EMT-related markers were detected. As demonstrated in Fig. 4A, the miR-137 mimic significantly attenuated the hypoxia-induced increases in the mRNA levels of $\mathrm{N}$-cadherin, vimentin and MMP2 compared with those in the $\mathrm{NC}+\mathrm{H}$ group. Western blot analysis revealed that compared with the $\mathrm{NC}+\mathrm{H}$ group, the miR-137 mimic down-regulated EMT-related protein expression levels, whereas the miR-137 inhibitor further up-regulated EMT-related protein expression in PC3 cells (Fig. 4B). In DU145 cells, the miR-137 mimic blocked the hypoxia-induced up-regulation of $\mathrm{N}$-cadherin, vimentin and 
A
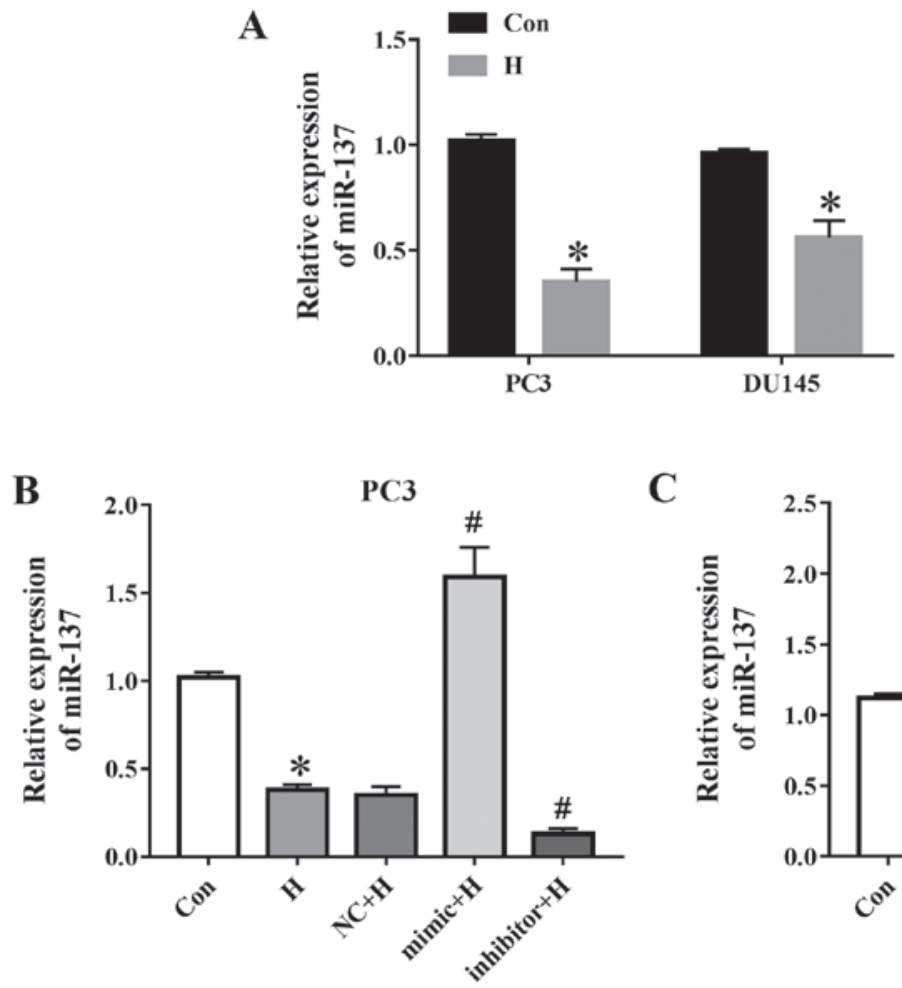

C

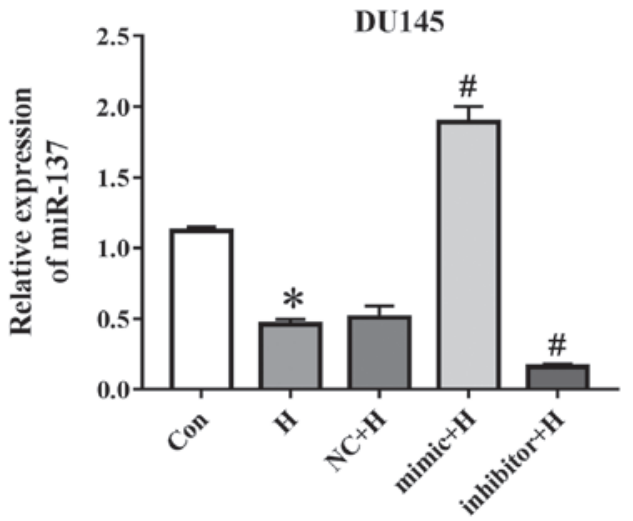

Figure 1. Hypoxia stimulates the up-regulation of miR-137. PC3 and DU145 cells were exposed to hypoxia ( $1 \% \mathrm{O}_{2}$ ) for $24 \mathrm{~h}$. (A) Expression of miR-137 was assessed by RT-qPCR. (B) PC3 and (C) DU145 cells were subjected to hypoxia after transfection with a miR-137 mimic or inhibitor. The mRNA level of miR-137 was determined. ${ }^{~} \mathrm{P}<0.05$ vs. Con; ${ }^{~} \mathrm{P}<0.05$ vs. H. miR, microRNA; Con, control; H, hypoxia; NC, miR-137 negative control; RT-qPCR, reverse transcription-quantitative PCR.
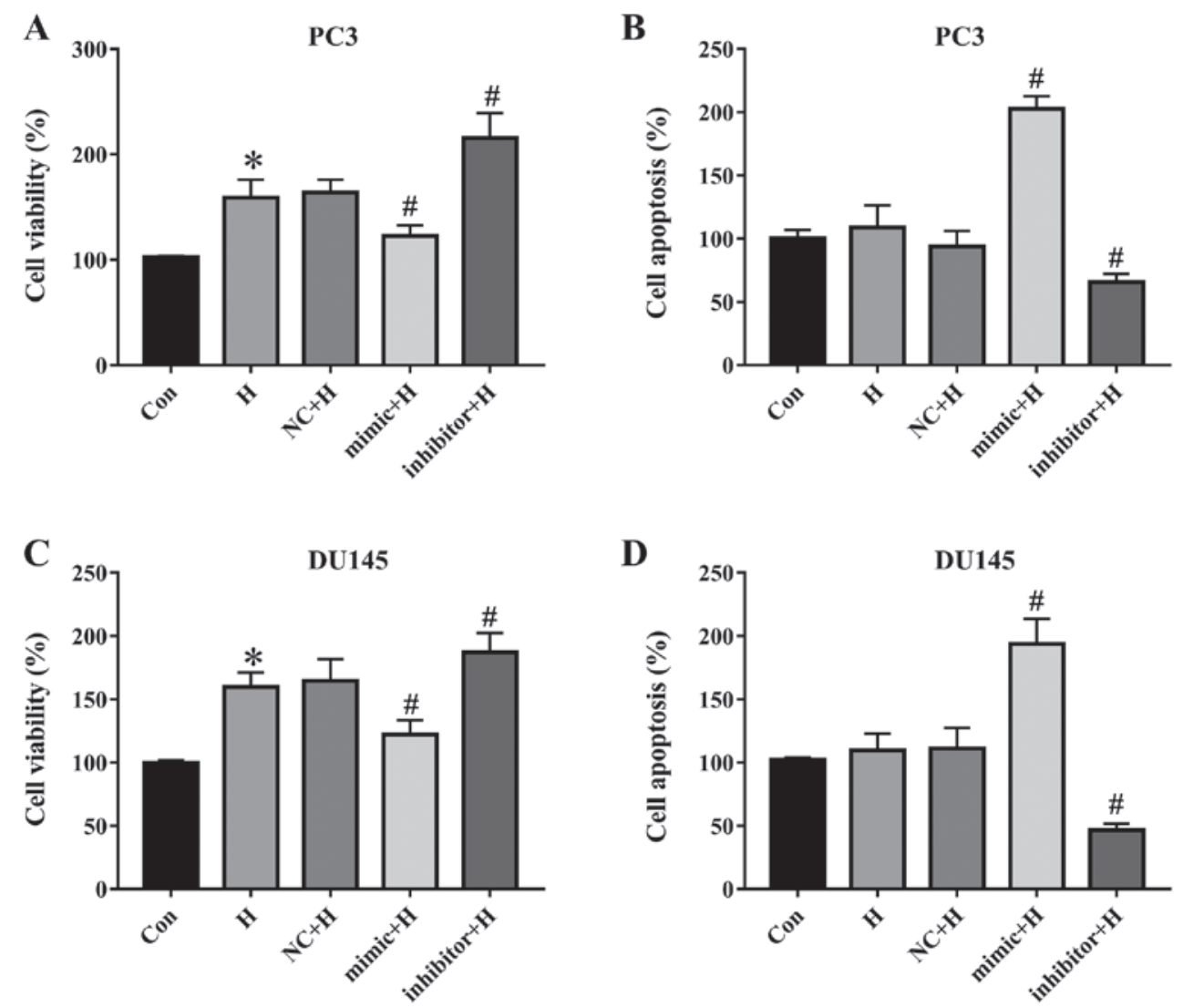

Figure 2. miR-137 regulates cell viability and apoptosis in prostate cancer cells. PC3 and DU145 cells were treated with a miR-137 mimic or inhibitor and subjected to hypoxia. (A) Cell viability and (B) apoptosis were determined using CCK-8 assay and Cell Death Detection ELISA plus kit, respectively, in PC3 cells. (C and D) CCK-8 assay and Cell Death Detection ELISA plus kit were performed to evaluate the (C) cell viability and (D) apoptosis of DU145 cells. ${ }^{*} \mathrm{P}<0.05$ vs. Con; ${ }^{~} \mathrm{P}<0.05$ vs. NC + H. miR, microRNA; Con, control; H, hypoxia; NC, miR-137 negative control; CCK-8, Cell Counting Kit-8. 
A

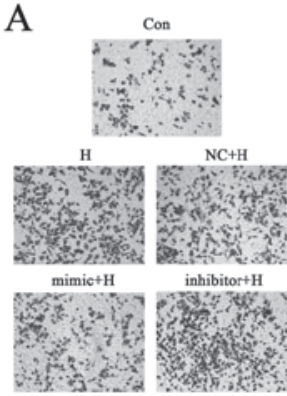

$\mathrm{C}$

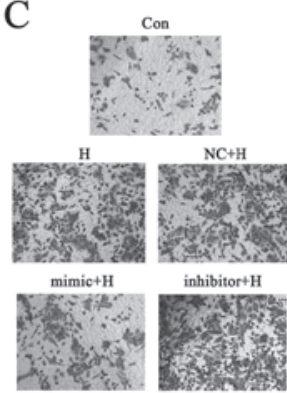

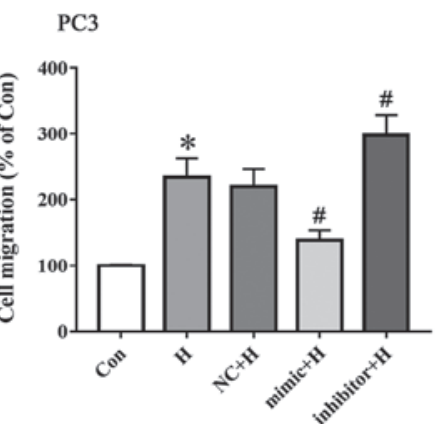

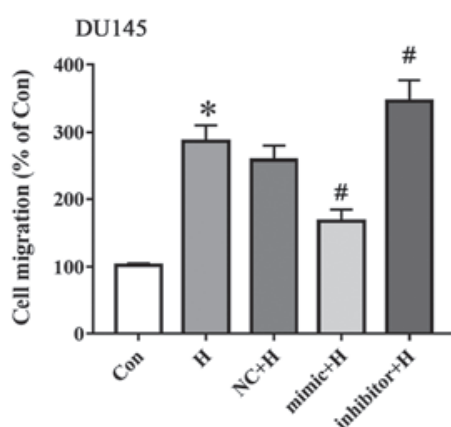

B

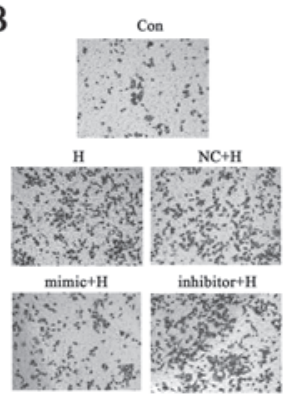

D

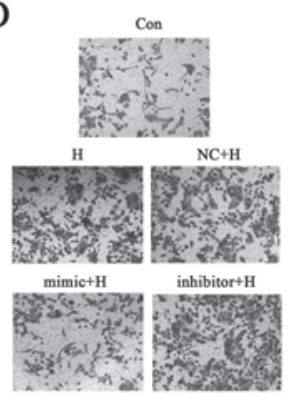

PC3

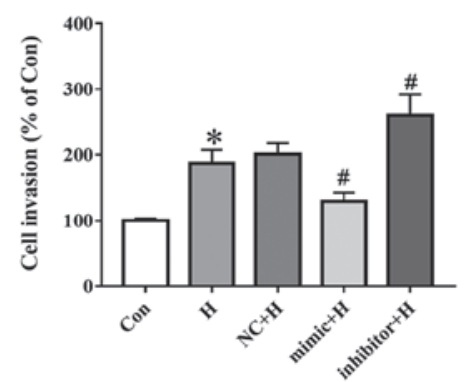

DU145

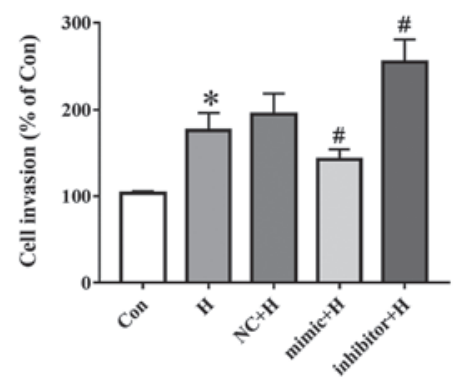

Figure 3. miR-137 inhibits cell migration and invasion induced by hypoxia in prostate cancer cells. PC3 and DU145 cells were transfected with a miR-137 mimic or inhibitor for $48 \mathrm{~h}$ and exposed to hypoxia. (A and B) Transwell assays were used to detect the PC3 cell (A) migration and (B) invasion. The (C) migration and (D) invasion of DU145 cells were detected by Transwell assays. "P<0.05 vs. Con; ${ }^{*} \mathrm{P}<0.05$ vs. NC $+\mathrm{H}$. miR, microRNA; Con, control; H, hypoxia; NC, miR-137 negative control.

MMP2 mRNA levels compared with the NC-transfected cells; however, ablation of miR-137 promoted hypoxia-induced increases in N-cadherin, vimentin, and MMP2 mRNA levels (Fig. 4C). In addition, the miR-137 mimic decreased the protein levels of EMT-related proteins, whereas the miR-137 inhibitor increased the protein levels of $\mathrm{N}$-cadherin, vimentin, and MMP2 in DU145 cells compared with those in the NC $+\mathrm{H}$ group (Fig. 4D). These results suggested that miR-137 inhibited the EMT in prostate cancer cells exposed to hypoxia.

miR-137 targets LGR4 in PC3 cells. Bioinformatics analysis identified LGR4 as a target gene of miR-137. The binding site between miR-137 and LGR4-wt or LGR4-mut is presented in Fig. 5A. The LGR4-3'-UTR was sequenced, and then nucleotide alignment was compared in NCBI. The similarity with NM_018490.5 was $99.09 \%$ (data not shown). To confirm this result, a dual-luciferase reporter gene assay was performed in PC3 cells after 48-h co-transfection with the miR-137 mimic or NC and LGR4-wt or LGR4-mut. As presented in Fig. 5B, luciferase activity decreased following co-transfection with LGR4-wt and the miR-137 mimic compared with that in the $\mathrm{NC}$ group, whereas no significant differences were observed in cells transfected with LGR4-mut. Additionally, compared with the $\mathrm{NC}+\mathrm{H}$ group, the miR-137 mimic negatively regulated the expression of LGR4 at the mRNA (Fig. 5C) and protein (Fig. 5D) levels under hypoxic conditions.

miR-137 regulates EGFR/ERK signaling by targeting LGR4. To elucidate the mechanism of miR-137 in the modulation of migration and EMT, PC3 cells were co-transfected with the miR-137 inhibitor and siLGR4 and subjected to hypoxia. The levels of LGR4 expression were decreased at the mRNA and protein levels following transfection with siLGR4 compare with that in the siCtrl group (Fig. S2). In addition, the miR-137 inhibitor elevated the LGR4 protein levels compared with those in the negative control group, whereas knockdown of LGR4 reduced the expression of LGR4 compared with that in the inhibitor + siCtrl group (Fig. 6A). Inhibition of miR-137 expression further up-regulated the hypoxia-mediated augmentation of EGFR phosphorylation compared with that in the $\mathrm{NC}+\mathrm{siCtrl}+\mathrm{H}$ group, whereas LGR4 silencing reversed this effect (Fig. 6B). Consistent with EGFR, the miR-137 inhibitor increased the phosphorylation of ERK compared with that in the negative control group, whereas LGR4 knockdown decreased it (Fig. 6C). These results suggested that miR-137 may regulate the EGFR/ERK signaling pathway by targeting LGR4.

miR-137 regulates $P C 3$ cell migration and EMT by targeting LGR4 via the EGFR/ERK signaling pathway. To examine the potential mechanisms of miR-137 in the regulation of the migration and EMT of $\mathrm{PC} 3$ cells, the cells were pretreated with AG1478, which is a selective inhibitor of EGFR. As presented in Fig. 7A and B, the miR-137 inhibitor increased cell migration and invasion under hypoxic conditions compared with the $\mathrm{NC}+\mathrm{H}$ group, whereas down-regulation of LGR 4 or disruption of the EGFR/ERK signaling pathway reversed these effects. Furthermore, the mRNA levels of N-cadherin, vimentin and MMP2 increased after transfection with the miR-137 inhibitor compared with those in the NC-transfected cells, but decreased following LGR4 knockdown or inhibition of the EGFR/ERK signaling pathway (Fig. 7C). Similarly, the effects of the miR-137 inhibitor on the regulation of $\mathrm{N}$-cadherin, vimentin 
A

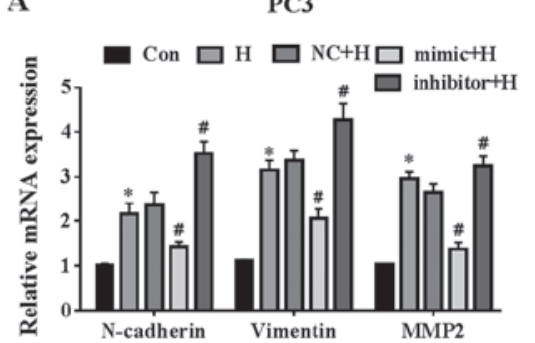

C

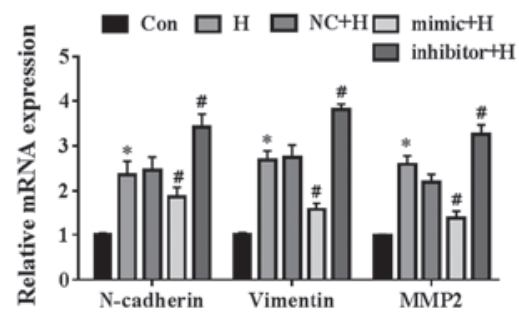

B

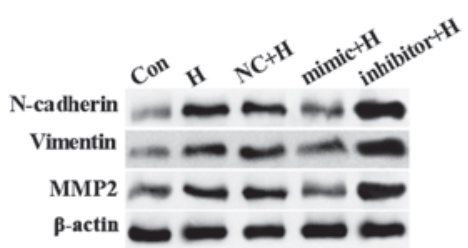

D

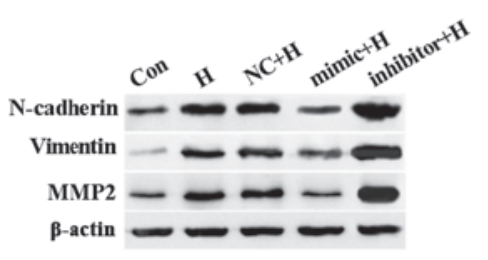

PC3

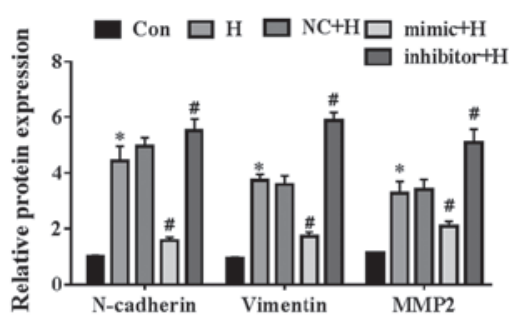

DU145

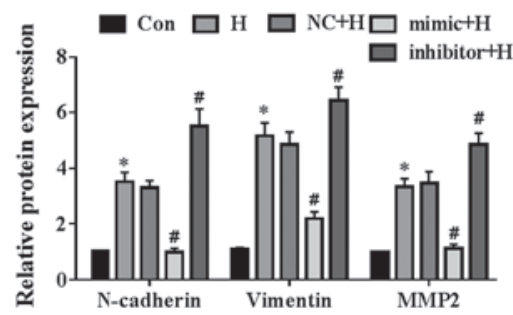

Figure 4. miR-137 inhibits hypoxia-induced epithelial-mesenchymal transition in prostate cancer cells. PC3 and DU145 cells were transfected with a miR-147 mimic or inhibitor for $48 \mathrm{~h}$ and exposed to hypoxia. (A and B) The N-cadherin, vimentin and MMP2 (A) mRNA, as well as (B) protein levels in PC3 cells were evaluated by RT-qPCR and western blotting, respectively. The (C) mRNA and (D) protein levels of N-cadherin, vimentin and MMP2 in DU145 cells were also tested by RT-qPCR and western blotting. "P<0.05 vs. Con; " $\mathrm{P}<0.05$ vs. NC + H. miR, microRNA; Con, control; H, hypoxia; NC, miR-137 negative control; RT-qPCR, reverse transcription-quantitative PCR; MMP2, matrix metalloproteinase 2.

A

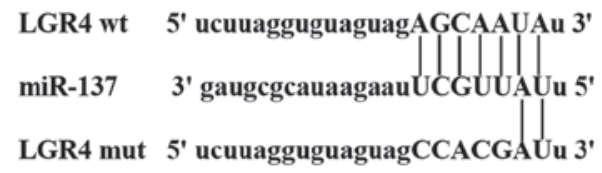

B

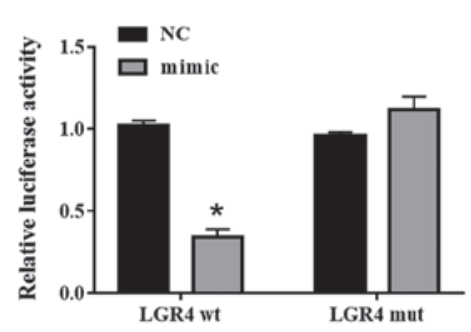

$\mathrm{C}$

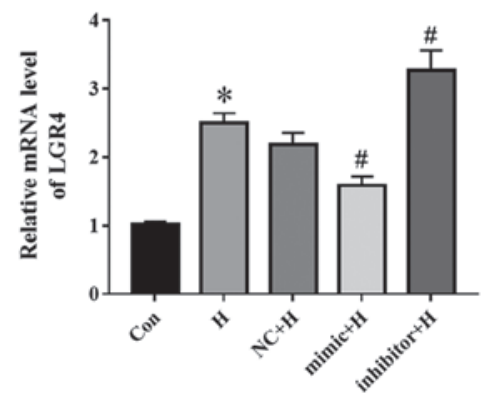

D
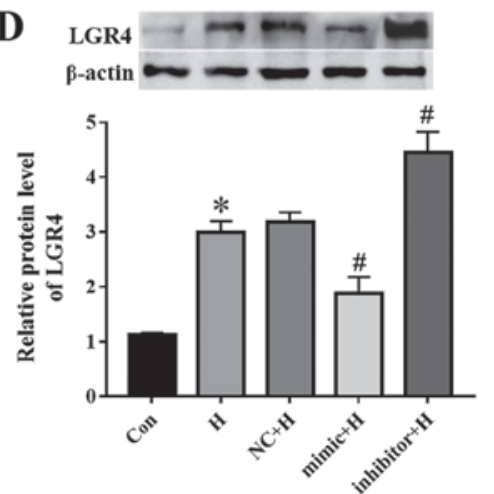

Figure 5. miR-137 directly targets LGR4 in PC3 cells. (A) The binding sites between miR-137 and LGR4-wt or LGR4-mut. (B) PC3 cells were co-transfected with NC or miR-137 mimic and LGR4-wt or with LGR4-mut for $48 \mathrm{~h}$, and relative luciferase activity was determined. The (C) mRNA and (D) protein levels of LGR4 were detected by RT-qPCR and western blotting, respectively. ${ }^{*} \mathrm{P}<0.05$ vs. Con or $\mathrm{NC}$; ${ }^{\mathrm{P}}<0.05 \mathrm{vs}$. NC $+\mathrm{H}$. miR, microRNA; Con, control; H, hypoxia; NC, miR-137 negative control; LGR4, leucine-rich repeat-containing G protein-coupled receptor 4; wt, wild-type; mut, mutant; RT-qPCR, reverse transcription-quantitative PCR.

and MMP2 protein levels were abolished by LGR4 silencing or EGFR/ERK signaling pathway inhibition in presence of hypoxia (Fig. 7D). These results suggested that miR-137 regulated the migration and EMT of PC3 cells by targeting LGR4 via the EGFR/ERK signaling pathway.

\section{Discussion}

Hypoxia is a common feature of solid tumors and a negative prognostic and predictive factor that contributes to tumor progression and chemoresistance (22). MicroRNAs are abnormally expressed when exposed to hypoxia, lipopolysaccharides or tumor necrosis factor- $\alpha(23,24)$. miR-137 is downregulated in the mouse brain during hypoxia (18). Consistent with these findings, the results of the present study demonstrated decreased levels of miR-137 in PC3 and DU145 cells after exposure to hypoxia, suggesting that miR-137 was involved in the hypoxia-mediated prostate cancer progression.

The responses of tumor cells to hypoxia include cell proliferation, differentiation and resistance to apoptosis (25). 
A

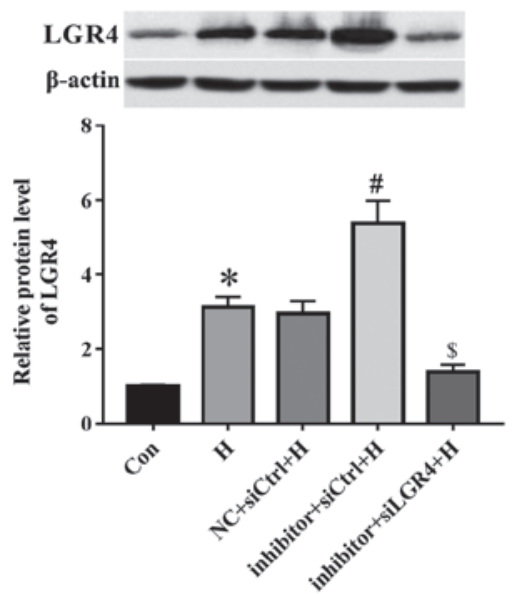

B

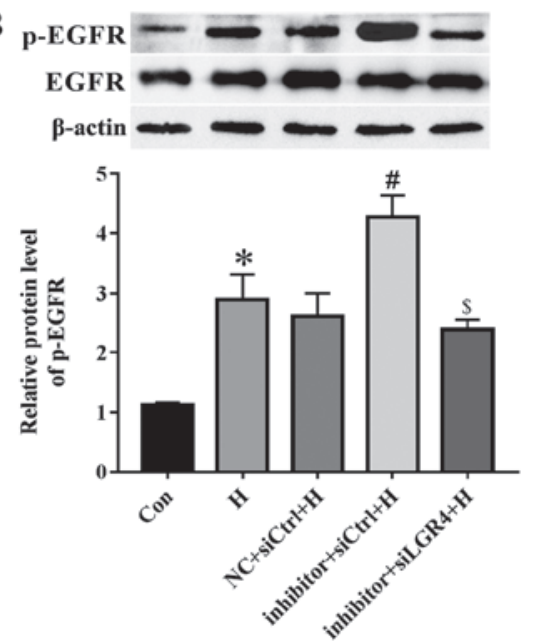

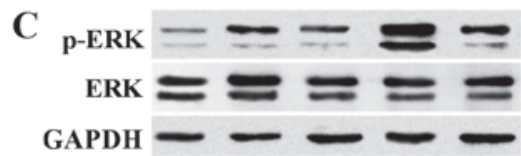

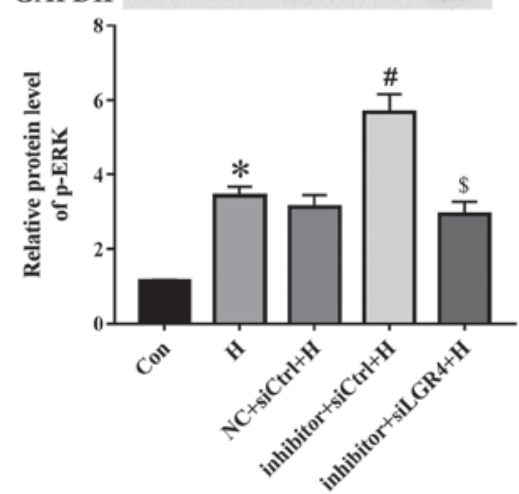

Figure 6. miR-137 regulates EGFR/ERK signaling by targeting LGR4. PC3 cells were co-transfected with the miR-137 inhibitor and siLGR4. (A) The expression of LGR4 was evaluated by western blotting. Phosphorylation levels of (B) EGFR and (C) ERK were tested by western blotting. "P<0.05 vs. Con; "P $<0.05$ vs. $\mathrm{NC}+\mathrm{H} ;{ }^{\mathrm{P}} \mathrm{P}<0.05$ vs. inhibitor+siCtrl+H. miR, microRNA; Con, control; H, hypoxia; NC, miR-137 negative control; si, small interfering RNA; LGR4, leucine-rich repeat-containing G protein-coupled receptor 4; siCtrl, negative control for siLGR4; EGFR, epidermal growth factor receptor.
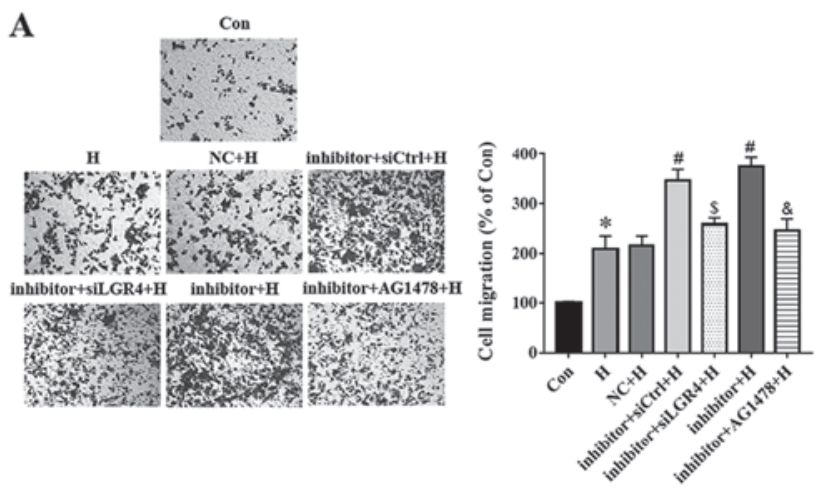

C

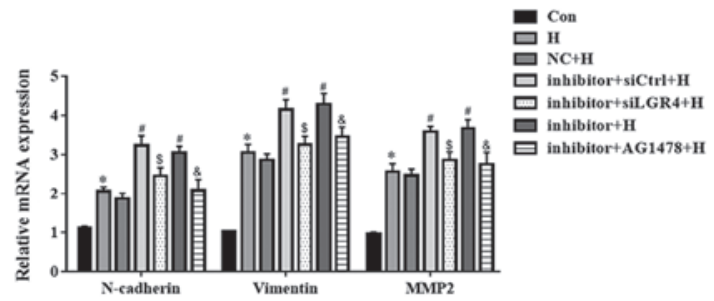

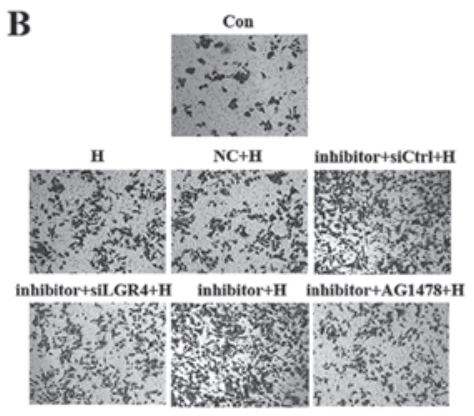

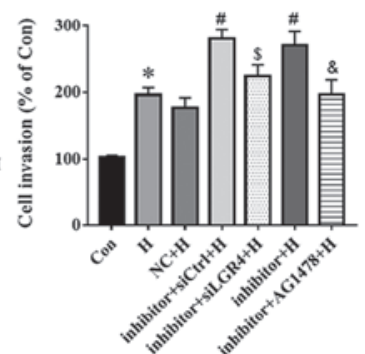

D

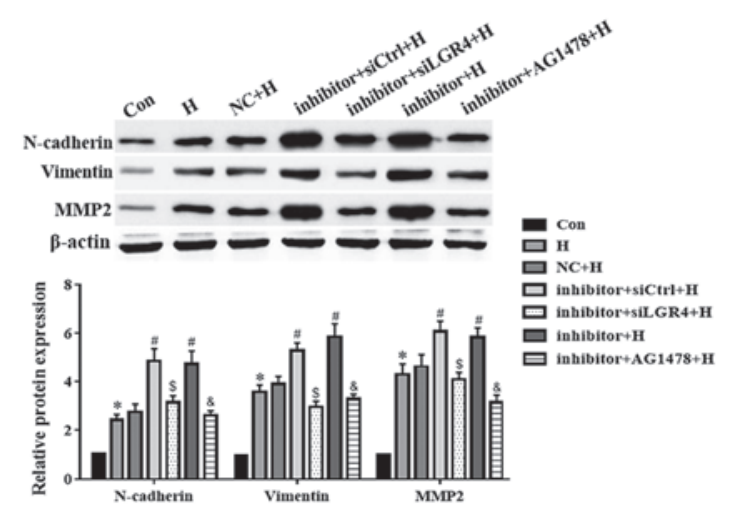

Figure 7. LGR4 silencing or EGFR/ERK blockade reverses the biological roles of miR-137 in PC3 cells. Cells were transfected with miR-137 inhibitor, and then treated with $100 \mathrm{nM}$ of AG1478 for $1 \mathrm{~h}$, and exposed to hypoxia. (A and B) A Transwell assay was used to evaluate the (A) migration and (B) invasion of PC3 cells. (C) The mRNA levels of N-cadherin, vimentin and MMP2 were analyzed by RT-qPCR. (D) The protein levels of N-cadherin, vimentin and MMP2 were detected using western blotting. ${ }^{*} \mathrm{P}<0.05$ vs. Con or $\mathrm{NC} ;{ }^{*} \mathrm{P}<0.05$ vs. $\mathrm{NC}+\mathrm{H} ;{ }^{\$} \mathrm{P}<0.05$ vs. inhibitor $+\mathrm{siCtrl}+\mathrm{H}$; ${ }^{\&} \mathrm{P}<0.05$ vs. inhibitor $+\mathrm{H}$. miR, microRNA; Con, control; H, hypoxia; NC, miR-137 negative control; si, small interfering RNA; LGR4, leucine-rich repeat-containing G protein-coupled receptor 4; siCtrl, negative control for siLGR4; EGFR, epidermal growth factor receptor; MMP2, matrix metalloproteinase 2; AG1478, a selective inhibitor of EGFR; RT-qPCR, reverse transcription-quantitative PCR.

The sensitivity to apoptosis is likely to be one of the critical factors in determining whether cancer cells survive (26). In the present study, hypoxia promoted cell survival, but had no effect on apoptosis in PC3 and DU145 cells. Coffey et al (27) previously demonstrated that PC3 and DU145 cells were resistant to apoptosis under hypoxic conditions, but LNCaP and PWR-1E cells were susceptible, which may be due to androgen-independent PC3 and DU145 expressing high levels of NLR family apoptosis inhibitory protein, cellular inhibitor of apoptosis 1 (cIAP-1) and cIAP-2, among which cIAP-2 
expression has been identified to be associated with apoptosis resistance. The results of the present study demonstrated that miR-137 prevented the proliferation and triggered the apoptosis of PC3 and DU145 cells. Consistent with these results, Huang et al (28) demonstrated that miR-137 overexpression reduced cell proliferation and increased apoptosis in acute lymphoblastic leukemia cells, which has also been observed in pancreatic cancer cells (29).

miR-137 is a suppressor of a number of tumors, including gastric cancer (30), hepatocellular (31) and renal cell (32) carcinoma. miR-137 attenuates the proliferation, migration, invasion and EMT of multiple types of cancer cells, including endometrial (33), colon (34) and triple-negative breast (35) cancer cells. Consistent with these findings, the results of the present study demonstrated that miR-137 reduced the hypoxia-induced cell viability, migration and invasion, as well as EMT-related protein expression of prostate cancer cells.

LGRs belong to the seven-transmembrane protein family with receptors for glycoprotein, thyrotropin, and luteinizing and follicle-stimulating hormones (36). LGR4, also termed G protein-coupled receptor 48, is closely associated with LGR5 and LGR6 (37). LGR4 serves a central role in the development of the male reproductive tract (38). LGR5 mostly occurs on the surface of gastrointestinal proliferative stem cells, where it serves as a specific molecular marker of stem cells (39). LGR6 appears on the surface of skin pluripotent stem cells (40). LGR4, LGR5 and LGR6 were initially considered to be orphan receptors until it was discovered that $\mathrm{R}$-spondin ligands directly linked these receptors to signal transduction in stem cells (41). Recently, LGR4 has been implicated in tumor progression, including papillary thyroid carcinoma (42) and prostate cancer (43).

The short-term survival rate in patients with high expression of LGR4 is lower compared with that in patients with low LGR4 expression, and LGR4 silencing inhibits the migration, invasion and scarring of the prostate cancer cell line DU145 (43). In addition, overexpression of LGR4 exacerbates tumorigenesis of prostate cancer cells $(44,45)$. PC3 and DU145 cells are commonly used to study prostate cancer progression due to high levels of migratory and invasive activity $(20,21)$. However, in all experiments in the present study, PC3 cells exhibited higher reproducibility of the results and more significant differences among groups compared with DU145 cells. Thus, PC3 cells were selected to study the association between miR-137 and LGR4, and the roles of LGR4 knockdown in hypoxia and miR-137 inhibitor-mediated cell migration, invasion and EMT-related protein expression. The results demonstrated that miR-137 directly targeted the 3'-UTR of LGR4 in PC3 cells. Of note, miR-137 negatively regulated the expression of LGR4, and LGR4 knockdown reversed the biological effects of the miR-137 inhibitor on PC3 cell migration and EMT, suggesting that miR-137 protected against hypoxia-mediated migration and EMT by inhibiting LGR4.

EGFR belongs to the receptor tyrosine kinase family and initiates tyrosine kinase activity after activation of EGF and other ligands, and further activates the downstream signal transduction pathways (46). Activation of EGFR/ERK signaling contributes to EMT and migration in prostate cancer, and LGR4 deficiency reduces phosphorylation of EGFR and
ERK. EGFR/ERK signaling is associated with tumorigenesis and is activated in lung cancer (47). EGFR/ERK signaling also inhibits cell proliferation and facilitates apoptosis in renal cell carcinoma (48). Blocking this pathway promotes cell death in prostate cancer (49). A previous report has demonstrated that LGR4 deficiency downregulates the phosphorylation of EGFR and ERK (50). The results of the present study demonstrated that miR-137 negatively regulated the EGFR/ERK signaling pathway by targeting LGR4 and that AG1478 abrogated the biological activities of miR-137. These results suggested that miR-137 regulated the EGFR/ERK signaling pathway by targeting LGR4 to impede the migration and EMT of prostate cancer cells.

In conclusion, the results of the present study revealed that hypoxia led to decreases in miR-137 expression. The miR-137 mimic inhibited, whereas the miR-137 inhibitor aggravated the hypoxia-induced cell migration, invasion and EMT in prostate cancer cells. Additionally, a luciferase reporter assay verified that miR-137 directly targeted LGR4. miR-137 negatively regulated EGFR/ERK by targeting LGR4. Knockdown of LGR4 abolished the effects of the miR-137 inhibitor on cell migration and EMT. AG1478 treatment also abrogated the effects of the miR-137 inhibitor on PC3 cell migration, invasion and EMT. Taken together, these results demonstrated that miR-137 may regulate the hypoxia-mediated migration and EMT in prostate cancer by targeting LGR4 via the EGFR/ERK signaling pathway. These results suggest a possible therapeutic strategy for prostate cancer treatment.

\section{Acknowledgements}

Not applicable.

\section{Funding}

This study was supported by The Medical Science and Technology Research Projects of Henan Province (grant no. 201602335).

\section{Availability of data and materials}

The datasets used and/or analyzed during the current study are available from the corresponding author on reasonable request.

\section{Authors' contributions}

HZ and FL conceived and designed the study. HZ, FL and JY designed the experiments. HZ, FL, JY, PL, JW, ZW, HL, DC and JD performed experiments and analyzed the data. HZ wrote the draft of manuscript. KZ and PD analyzed the data and revised the paper. All authors have read and approved the final manuscript.

\section{Ethics approval and consent to participate}

Not applicable.

\section{Patient consent for publication}

Not applicable. 


\section{Competing interests}

The authors declare that they have no competing interests.

\section{References}

1. Bashir MN: Epidemiology of prostate cancer. Asian Pac J Cancer Prev 16: 5137-5141, 2015 .

2. Kazuto I: Prostate cancer in Asian men. Nat Rev Urol 11: 197-212, 2014

3. Zhu Y, Freedland SJ and Ye D: Prostate cancer and prostatic diseases best of Asia, 2019: Challenges and opportunities. Prostate Cancer Prostatic Dis: Dec 6, 2019 (Epub ahead of print).

4. Marusic G, Vojinov S and Levakov I: Treatment of locally advanced prostatic cancer. Med Pregl 63: 689-695, 2010 (In Serbian).

5. Noman MZ, Hasmim M, Messai Y, Terry S, Kieda C, Janji B and Chouaib S: Hypoxia: A key player in antitumor immune response. A review in the theme: Cellular responses to hypoxia Am J Physiol Cell Physiol 309: C569-C579, 2015.

6. Ramteke A, Ting H, Agarwal C, Mateen S, Somasagara R, Hussain A, Graner M, Frederick B, Agarwal R and Deep G: Exosomes secreted under hypoxia enhance invasiveness and stemness of prostate cancer cells by targeting adherens junction molecules. Mol Carcinog 54: 554-565, 2015.

7. Deep G and Panigrahi GK: Hypoxia-induced signaling promotes prostate cancer progression: Exosomes role as messenger of hypoxic response in tumor microenvironment. Crit Rev Oncog 20: 419-434, 2015.

8. Roldo C, Missiaglia E, Hagan JP, Falconi M, Capelli P, Bersani S, Calin GA, Volinia S, Liu CG, Scarpa A and Croce CM: MicroRNA expression abnormalities in pancreatic endocrine and acinar tumors are associated with distinctive pathologic features and clinical behavior. J Clin Oncol 24: 4677-4684, 2006

9. Farazi TA, Hoell JI, Morozov P and Tuschl T: MicroRNAs in human cancer. Adv Exp Med Biol 774: 1-20, 2013.

10. Hong X and Yu JJ: MicroRNA-150 suppresses epithelial mesenchymal transition, invasion and metastasis in prostate cancer through the TRPM4-mediated $\beta$-catenin signaling pathway. Am J Physiol Cell Physiol 316: C463-C480, 2019.

11. Blenkiron C, Goldstein LD, Thorne NP, Spiteri I, Chin SF Dunning MJ, Barbosa-Morais NL, Teschendorff AE, Green AR, Ellis IO, et al: MicroRNA expression profiling of human breast cancer identifies new markers of tumor subtype. Genome Biol 8: R214, 2007.

12. Kota J, Chivukula RR, O'Donnell KA, Wentzel EA, Montgomery CL, Hwang HW, Chang TC, Vivekanandan P, Torbenson M, Clark KR, et al: Therapeutic microRNA delivery suppresses tumorigenesis in a murine liver cancer model. Cell 137: 1005-1017, 2009.

13. Zhao S, Gao X, Zang S, Li Y, Feng X and Yuan X: MicroRNA-383-5p acts as a prognostic marker and inhibitor of cell proliferation in lung adenocarcinoma by cancerous inhibitor of protein phosphatase 2A. Oncol Lett 14: 3573-3579, 2017.

14. Zhiping C, Shijun T, Linhui W, Yapei W, Lianxi Q and Qiang D: miR-181a promotes epithelial to mesenchymal transition of prostate cancer cells by targeting TGIF2. Eur Rev Med Pharmacol Sci 21: 4835-4843, 2017.

15. Yang ZG, Ma XD, He ZH and Guo YX: miR-483-5p promotes prostate cancer cell proliferation and invasion by targeting RBM5. Int Braz J Urol 43: 1060-1067, 2017.

16. Xu S, Ge J, Zhang Z and Zhou W: miR-129 inhibits cell proliferation and metastasis by targeting ETS1 via PI3K/AKT/mTOR pathway in prostate cancer. Biomed Pharmacother 96: 634-641, 2017.

17. Pashaei E, Ahmady M, Ozen M and Aydin N: Meta-analysis of miRNA expression profiles for prostate cancer recurrence following radical prostatectomy. PLoS One 12: e0179543, 2017.

18. Li W, Zhang X, Zhuang H, Chen HG, Chen Y, Tian W, Wu W, Li Y, Wang S, Zhang L, et al: MicroRNA-137 is a novel hypoxia-responsive microRNA that inhibits mitophagy via regulation of two mitophagy receptors FUNDC1 and NIX. J Biol Chem 289: 10691-10701, 2014.

19. Livak KJ and Schmittgen TD: Analysis of relative gene expression data using real-time quantitative PCR and the 2(-Delta Delta C(T)) method. Methods 25: 402-408, 2001
20. Pulukuri SM, Gondi CS, Lakka SS, Jutla A, Estes N, Gujrati M and Rao JS: RNA interference-directed knockdown of urokinase plasminogen activator and urokinase plasminogen activator receptor inhibits prostate cancer cell invasion, survival, and tumorigenicity in vivo. J Biol Chem 280: 36529-36540, 2005.

21. Viticchie G, Lena AM, Latina A, Formosa A, Gregersen LH, Lund AH, Bernardini S, Mauriello A, Miano R, Spagnoli LG, et al: miR-203 controls proliferation, migration and invasive potential of prostate cancer cell lines. Cell Cycle 10: 1121-1131, 2011.

22. Vaupel $P$ and Mayer A: Hypoxia in cancer: Significance and impact on clinical outcome. Cancer Metastasis Rev 26: 225-239, 2007.

23. Shin S, Moon KC, Park KU and Ha E: MicroRNA-513a-5p mediates TNF- $\alpha$ and LPS induced apoptosis via downregulation of X-linked inhibitor of apoptotic protein in endothelial cells. Biochimie 94: 1431-1436, 2012.

24. Chan SY, Zhang YY, Hemann C, Mahoney CE, Zweier JL and Loscalzo J: MicroRNA-210 controls mitochondrial metabolism during hypoxia by repressing the iron-sulfur cluster assembly proteins ISCU1/2. Cell Metab 10: 273-284, 2009.

25. Tang J, Chen Y, Cui R, Li D, Xiao L, Lin P, Du Y, Sun H, Yu X and Zheng X: Upregulation of fractalkine contributes to the proliferative response of prostate cancer cells to hypoxia via promoting the G1/S phase transition. Mol Med Rep 12: 7907-7914, 2015.

26. Shannon AM, Bouchier-Hayes DJ, Condron CM and Toomey D: Tumour hypoxia, chemotherapeutic resistance and hypoxia-related therapies. Cancer Treat Rev 29: 297-307, 2003.

27. Coffey RN, Morrissey C, Taylor CT, Fitzpatrick JM and Watson RW: Resistance to caspase-dependent, hypoxia-induced apoptosis is not hypoxia-inducible factor-1 alpha mediated in prostate carcinoma cells. Cancer 103: 1363-1374, 2005.

28. Huang Y,Zou Y,Zheng R and Ma X: miR-137 inhibits cell proliferation in acute lymphoblastic leukemia by targeting JARID1B. Eur J Haematol 103: 215-224, 2019.

29. Ding F, Zhang S, Gao S, Shang J, Li Y, Cui N and Zhao Q: miR-137 functions as a tumor suppressor in pancreatic cancer by targeting MRGBP. J Cell Biochem 119: 4799-4807, 2018.

30. Du Y, Chen Y, Wang F and Gu L: miR-137 plays tumor suppressor roles in gastric cancer cell lines by targeting KLF12 and MYO1C. Tumour Biol 37: 13557-13569, 2016.

31. Huang B, Huang M and Li Q: miR-137 suppresses migration and invasion by targeting EZH2-STAT3 signaling in human hepatocellular carcinoma. Pathol Res Pract 214: 1980-1986, 2018.

32. Wang M, Gao H, Qu H, Li J, Liu K and Han Z: miR-137 suppresses tumor growth and metastasis in clear cell renal cell carcinoma. Pharmacol Rep 70: 963-971, 2018.

33. Zhang W, Chen JH, Shan T, Aguilera-Barrantes I, Wang LS, Huang TH, Rader JS, Sheng X and Huang YW: miR-137 is a tumor suppressor in endometrial cancer and is repressed by DNA hypermethylation. Lab Invest 98: 1397-1407, 2018.

34. Bi WP, Xia M and Wang XJ: miR-137 suppresses proliferation, migration and invasion of colon cancer cell lines by targeting TCF4. Oncol Lett 15: 8744-8748, 2018.

35. Lee SJ, Jeong JH, Kang SH, Kang J, Kim EA, Lee J, Jung JH, Park HY and Chae YS: MicroRNA-137 inhibits cancer progression by targeting Del-1 in Triple-negative breast cancer cells. Int J Mol Sci 20: pii: E6162, 2019.

36. Schoore GV, Mendive F, Pochet R and Vassart G: Expression pattern of the orphan receptor LGR4/GPR48 gene in the mouse. Histochem Cell Biol 124: 35-50, 2005

37. Carmon KS, Gong X, Lin Q, Thomas A and Liu Q: R-spondins function as ligands of the orphan receptors LGR4 and LGR5 to regulate Wnt/beta-catenin signaling. Proc Natl Acad Sci USA 108: 11452-11457, 2011.

38. Hoshii $\mathrm{T}$, Takeo $\mathrm{T}$, Nakagata $\mathrm{N}$, Takeya $\mathrm{M}$, Araki $\mathrm{K}$ and Yamamura K: LGR4 regulates the postnatal development and integrity of male reproductive tracts in mice. Biol Reprod 76: 303-313, 2007.

39. Koo BK and Clevers H: Stem cells marked by the R-spondin receptor LGR5. Gastroenterology 147: 289-302, 2014.

40. Leushacke M and Barker N: Lgr5 and Lgr6 as markers to study adult stem cell roles in self-renewal and cancer. Oncogene 31: 3009-3022, 2012

41. Dongli W, Binlu H, Senyan Z, Xiaojuan Y, Wei W and Xinquan W: Structural basis for R-spondin recognition by LGR4/5/6 receptors. Genes Dev 27: 1339-1344, 2013.

42. Kang YE, Kim JM, Kim KS, Chang JY, Jung M, Lee J, Yi S, Kim HW, Kim JT, Lee K, et al: Upregulation of RSPO2-GPR48/LGR4 signaling in papillary thyroid carcinoma contributes to tumor progression. Oncotarget 8: 114980-114994, 2017. 
43. Luo W, Tan P, Rodriguez M, He L, Tan K, Zeng L, Siwko S and Liu M: Leucine-rich repeat-containing $G$ protein coupled receptor $4(\mathrm{Lgr} 4)$ is necessary for prostate cancer metastasis via epithelial-mesenchymal transition. J Biol Chem 292: 15525-15537, 2017.

44. Zhang J, Qi L, Zhang S, Xu Q and Wang T: Lgr4 promotes prostate tumorigenesis through the Jmjd2a/AR signaling pathway. Exp Cell Res 349: 77-84, 2016.

45. Fang L, Junmin Y, Junyong W, Lijuan Z, Rui F, Hao Z and Qingsong Z: GPCR48/LGR4 promotes tumorigenesis of prostate cancer via PI3K/Akt signaling pathway. Med Oncol 32: 49, 2015.

46. Orton RJ, Adriaens ME, Gormand A, Sturm OE, Kolch W and Gilbert DR: Computational modelling of cancerous mutations in the EGFR/ERK signalling pathway. BMC Syst Biol 3: 100, 2009.

47. Yang Y, Zhao W, Xu QW, Wang XS, Zhang Y and Zhang J: IQGAP3 promotes EGFR-ERK signaling and the growth and metastasis of lung cancer cells. PLoS One 9: e97578, 2014.
48. Li L, Gao Y, Zhang L, Zeng J, He D and Sun Y: Silibinin inhibits cell growth and induces apoptosis by caspase activation, down-regulating survivin and blocking EGFR-ERK activation in renal cell carcinoma. Cancer Lett 272: 61-69, 2008.

49. Oh HY, Lee EJ, Yoon S, Chung BH, Cho KS and Hong SJ: Cholesterol level of lipid raft microdomains regulates apoptotic cell death in prostate cancer cells through EGFR-mediated Akt and ERK signal transduction. Prostate 67: 1061-1069, 2010.

50. Pan H, Cui H, Liu S, Qian Y, Wu H, Li L, Guan Y, Guan X, Zhang L, Fan HY, et al: Lgr4 gene regulates corpus luteum maturation through modulation of the WNT-mediated EGFR-ERK signaling pathway. Endocrinology 155: 3624-3637, 2014. 